\title{
Expression of neuronal nitric oxide synthase in the internal thoracic artery and saphenous vein
}

George D. Webb, PhD, a,b,c,* Lay Har Lim, BSc, ${ }^{a, *}$ Vernon M. S. Oh, MD, ${ }^{c, *}$ Reida El Oakley, MD, ${ }^{d, *}$ Chuen Neng Lee, MD, ${ }^{d, *}$ Poo Sing Wong, MD, ${ }^{d, *}$ W. Maung Maung Aye, MD, ${ }^{d}$ Edwin S. Y. Chan, PhD, ${ }^{e}$ and Philip K. Moore, PhD ${ }^{a, *}$

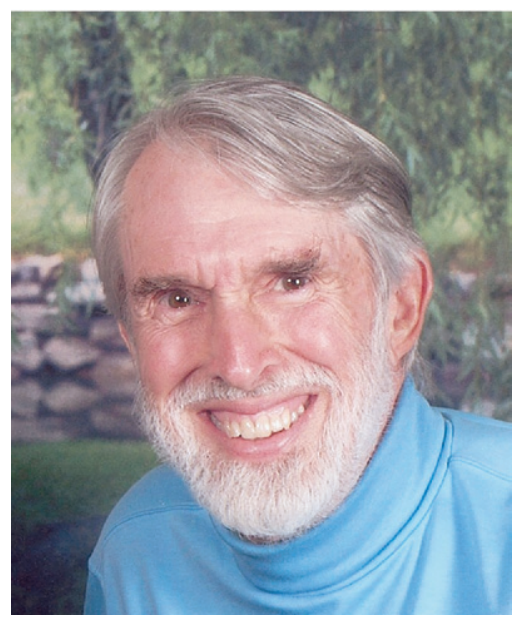

Dr Webb

Objectives: Endothelial nitric oxide synthase (type III) generates nitric oxide, which dilates blood vessels. Recently, it was discovered that arterial smooth muscle cells express neuronal nitric oxide synthase (type I). The purpose of this study was to determine the relative amounts of neuronal nitric oxide synthase in the human internal thoracic artery and saphenous vein.

Methods: Remainder segments of internal thoracic arteries and saphenous veins were obtained from 45 patients during coronary artery bypass grafting. Western blotting used specific antibodies against the 3 isoforms of human nitric oxide synthase and $\beta$-actin (for normalization) to measure the relative amounts of the 3 isoforms of nitric oxide synthase proteins in vessel specimens. Immunohistochemistry was used to localize the 3 proteins in specific cells.

Results: Western blotting detected all 3 isoforms of nitric oxide synthase in the human internal thoracic artery. The band density (normalized to $\beta$-actin) of neuronal nitric oxide synthase was not significantly different from the band density of endothelial nitric oxide synthase. The amounts of neuronal nitric oxide synthase in arteries and veins were equal. Immunohistochemistry showed that the highest expression of endothelial nitric oxide synthase was in endothelial cells, but some expression was also seen in smooth muscle cells. Most of the neuronal nitric oxide synthase was in smooth muscle cells. The location and relative amounts of inducible nitric oxide synthase were variable.

Medicine $^{\mathrm{c}}$ and Surgery, ${ }^{\mathrm{d}}$ National University of Singapore, Singapore; the Department of Molecular Physiology and Biophysics, ${ }^{\text {b }}$ University of Vermont, Burlington, Vt; and the Clinical Trials and Epidemiology Research Unit, ${ }^{\text {e }}$ Singapore

Supported by the Singapore National Medical Research Council (grant no. NMRC/ 0402/2000), the Singapore National Healthcare Group (grant no. NHG-RPR/02017), and the Office of Life Science of the National University of Singapore (grant no. R-184-000-074-712).

Received for publication Feb 8, 2006; revisions received July 24, 2006; accepted for publication Aug 3, 2006.

Address for reprints: George D. Webb, $\mathrm{PhD}$, Department of Molecular Physiology and Biophysics, University of Vermont, College of Medicine, HSRF Building, Burlington, VT 05405 (E-mail webb@ physiology.med.uvm.edu).

*Members of the Cardiovascular Biology Research Group, National University of Singapore.

J Thorac Cardiovasc Surg 2006;132:1131-6

$0022-5223 / \$ 32.00$

Copyright (C) 2006 by The American Association for Thoracic Surgery

doi:10.1016/j.jtcvs.2006.08.001

Conclusions: Neuronal nitric oxide synthase is expressed in the vascular smooth muscle of patients undergoing bypass, and the amount in the internal thoracic artery is the same as in the saphenous vein.

I n 1980, it was discovered that the endothelium of blood vessels releases a substance that acts on the underlying smooth muscle, causing it to relax. ${ }^{1}$ In 1987, this vasodilator substance was shown to be nitric oxide (NO). ${ }^{2}$ The $\mathrm{NO}$ is generated from L-arginine by the enzyme nitric oxide synthase (NOS). Three isoforms of NOS have been described: neuronal NOS (nNOS; type I), inducible NOS (iNOS; type II), and endothelial NOS (eNOS; type III). ${ }^{3,4}$ It is commonly thought that the major enzyme catalyzing the formation of NO in blood vessels is eNOS, which is located in endothelial cells. Recently, however, an article in the Journal provided evidence that there is about as much eNOS in the smooth muscle cells as there is in endothelial cells, the human internal thoracic artery (ITA), and the radial artery but not in the saphenous vein (SV). ${ }^{5}$ It was suggested that the eNOS expressed in the smooth muscle cells of the ITA is important for helping to prevent atherosclerosis and that it might account for the longer life of ITA grafts compared with SV grafts. ${ }^{5}$

It has also been reported that nNOS is expressed in the smooth muscle cells of some rat $^{6}$ and human ${ }^{7}$ arteries. The authors of the study that included human arteries suggested that the NO produced by NOS in the smooth muscle cells might modulate arterial function independently of NO released from endothelial cells. ${ }^{7}$ We do not know of any 


\author{
Abbreviations and Acronyms \\ eNOS = endothelial nitric oxide synthase \\ iNOS = inducible nitric oxide synthase \\ ITA $=$ internal thoracic artery \\ nNOS = neuronal nitric oxide synthase \\ $\mathrm{NO}=$ nitric oxide \\ NOS = nitric oxide synthase \\ PBS = phosphate-buffered saline \\ $\mathrm{RT}-\mathrm{PCR}=$ reverse transcription-polymerase chain \\ reaction \\ SV = saphenous vein
}

studies showing the expression of nNOS in the human ITA or SV. Thus we undertook this study to see whether and where nNOS might be expressed in the ITA or SV and to test the hypothesis that there is more nNOS in the ITA than in the SV, which could provide an additional reason for the longevity of ITA grafts. In the present experiments we used Western blotting to compare the amounts of eNOS, nNOS, and iNOS in the ITA and to compare the amount of nNOS in the ITA with the amount in the SV. We also localized each of the 3 isoforms of NOS in the ITA and SV by using immunohistochemistry.

\section{Methods}

\section{Arteries and Veins}

This study was approved by the Institutional Review Board of the National University Hospital, National Healthcare Group, Singapore, and the Institutional Review Board of the National University of Singapore. Informed patient consent was obtained before surgical intervention. Remainder segments of ITAs and sometimes also SVs were collected during coronary artery bypass grafting. Blood vessels were collected from 45 patients in this study. The patients were $80 \%$ men, the average age was 60 years, $73 \%$ had type II diabetes, $16 \%$ had type I diabetes, and $11 \%$ were nondiabetic. The patients for whom Western blot data are presented were all being treated for hypertension: $88 \%$ were taking $\beta$-blockers, $59 \%$ were taking angiotensin-converting enzyme inhibitors, and $12 \%$ were taking calcium-channel blockers. Eighty-two percent had hyperlipidemia, and 59\% had a history of smoking.

Immediately after the surgeon cut off the remainder segment of the ITA, the adhering connective tissue was carefully removed from the surface (requiring 5 minutes). The cleaned remainder segment, usually about $10 \mathrm{~mm}$ long, was usually cut into shorter segments. A 2-mm segment was rinsed in phosphate-buffered saline (PBS; $8 \mathrm{~g} / \mathrm{L}$ $\mathrm{NaCl}, 0.2 \mathrm{~g} / \mathrm{L} \mathrm{KCl}, 1.44 \mathrm{~g} / \mathrm{L} \mathrm{Na}_{2} \mathrm{HPO}_{4}$, and $\left.0.24 \mathrm{~g} / \mathrm{L} \mathrm{KH}_{2} \mathrm{PO}_{4}\right)$ and immersed in buffered $10 \%$ (vol/vol) formalin for subsequent paraffin embedding and immunohistochemistry. A 3-mm segment was rinsed in PBS, immediately snap-frozen in liquid nitrogen, and stored at $-80^{\circ} \mathrm{C}$ before protein extraction for Western blotting. The remaining segment was immersed in RNAlater reagent (Qiagen) for subsequent RNA extraction and reverse transcription-polymerase chain reaction (RT-PCR). A remainder segment of the SV was collected from some of the patients. We did not need to clean it because the assisting surgeon had already removed the adhering connective tissue. The SV had been gently distended with heparinized blood. Histologic sections usually showed an intact endothelium.

\section{Measurement of NOS mRNA With RT-PCR}

Total RNA was extracted with Trizol and reverse transcribed to cDNA. Conventional polymerase chain reaction was carried out on the cDNA by using primers that were custom made for us by Research Biolabs, Singapore. For the nNOS PCR (Figure 1, A), we used the following primer pair: forward, GTACCGAGCCCTGAAGGAGC; reverse, GGTTGTCATCCCTCATCCG. We used up the arteries from many of the patients in the early phase of this study while attempting to obtain quantitative results with real-time RT-PCR. We tested many NOS primers, some previously used in other laboratories, and many we designed with Gene Runner software, but none of them yielded quantitatively reliable real-time RT-PCR results. The reason was that we could not find primers that were both isoform specific and did not have problematic primer dimer formation because of the high GC content and the high degree of identities among the 3 NOS cDNAs.

\section{Measurement of NOS Proteins With Western Blotting}

Total protein was extracted from the frozen human blood vessels by pulverizing them with a mortar and pestle under liquid nitrogen, together with modified RIPA buffer containing freshly added protease inhibitor cocktail (Sigma Aldrich, St. Louis, Mo). Protein extracts were stored at $-80^{\circ} \mathrm{C}$ in small aliquots to avoid repeated freeze-thaw cycles, which degrade the NOS proteins. Standard sodium dodecylsulfate-polyacrylamide gel electrophoresis separation of the extracted proteins with $7.5 \%$ polyacrylamide precast gels (Bio-Rad) was carried out. Gels were Western blotted onto polyvinylidene difluoride membranes, and the blots were incubated overnight at $4{ }^{\circ} \mathrm{C}$ with anti-NOS or anti- $\beta$-actin antibodies. Specific antibodies against each of the 3 isoforms of human NOS were purchased from BD Transduction Labs, BD Biosciences, Singapore Distributor. We visualized the bands on the Western blots by means of chemiluminescence with ECL Western Blotting Detection Reagents (GE Healthcare, Chalford St. Giles, UK). Band density was measured with Quantity One (BioRad) software. The NOS band density was normalized to the $\beta$-actin band in the same lane by dividing the NOS density by the $\beta$-actin density.

\section{Localization of NOS Proteins in Histologic Sections}

Immunohistochemistry was used to identify cells expressing each of the 3 isoforms of NOS protein in paraffin sections of arteries and veins. We blocked endogenous peroxidase in the $5-\mu \mathrm{m}$ sections with $3 \% \mathrm{vol} / \mathrm{vol} \mathrm{H}_{2} \mathrm{O}_{2}$ in methanol and retrieved antigen by heating almost to boiling in $\mathrm{pH} 6$ sodium citrate buffer. Nonspecific sites were blocked with $2.5 \%$ bovine serum albumin plus $10 \%$ horse or goat (depending on which secondary antibody was being used) serum. The PBS used throughout contained $0.2 \% \mathrm{vol} / \mathrm{vol}$ Tween 20 and, except for the blocking solution above, also contained $2.5 \% \mathrm{wt} / \mathrm{vol}$ bovine serum albumin and $4 \% \mathrm{vol} / \mathrm{vol}$ horse or goat serum. Sections were incubated overnight at $4{ }^{\circ} \mathrm{C}$ in PBS containing one of the primary antibodies. In addition to using the same antibodies from BD Transduction Labs as were used for Western blotting, we also used primary antibodies from Santa Cruz Biotechnology. Antibodies from these 2 sources produced similar results. After incubation with primary antibody, we used proce- 

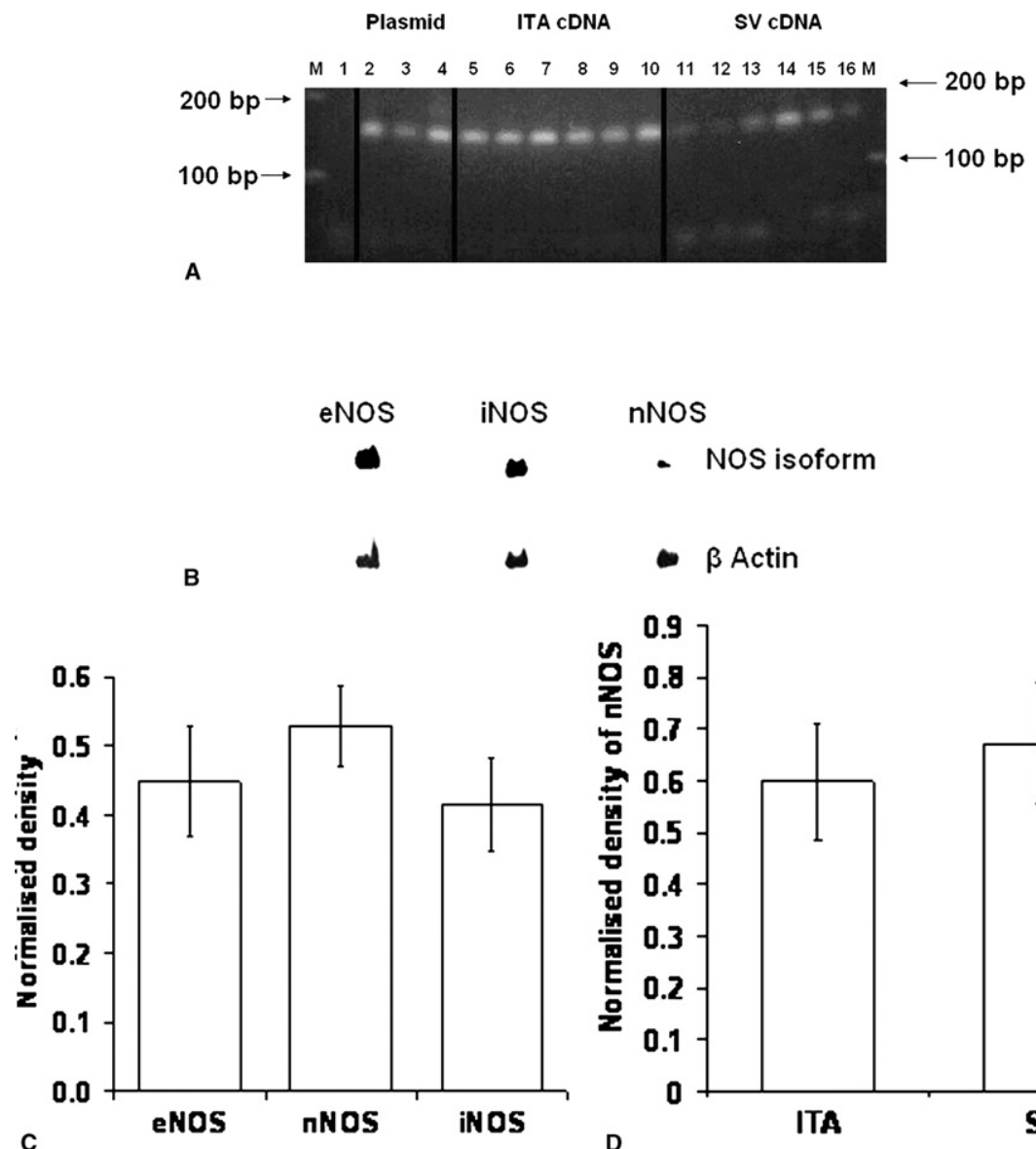

C
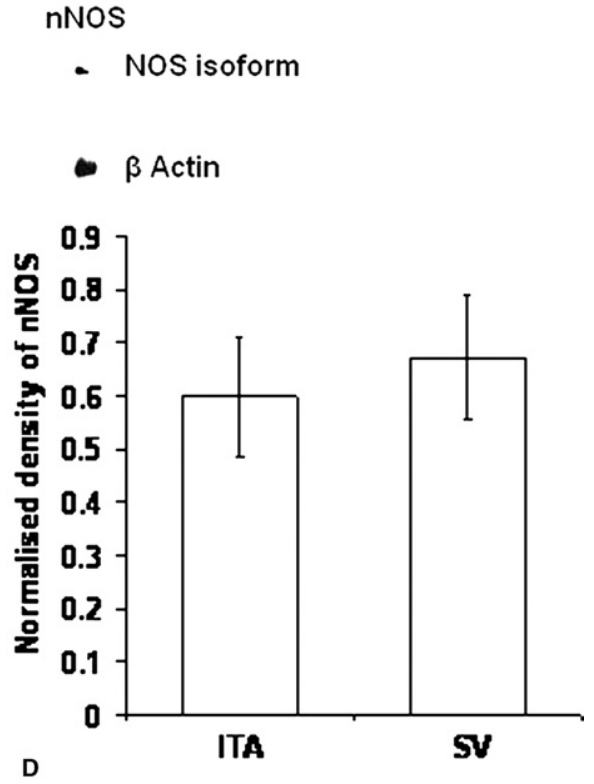

Figure 1. Reverse transcription-polymerase chain reaction (RT-PCR) and Western blot results. A, Agarose gel of RT-PCR products. Primers for neuronal nitric oxide synthase (nNOS), which produce a 161-bp product, were used. Lane 1, Negative control; lanes 2 and 3, nNOS cDNA standard, 8 copies per tube; lane 4, 800 copies; lanes 5-10, internal thoracic artery (ITA) cDNA (2 concentrations); lanes 11-16, saphenous vein ( $S V$; 2 concentrations). B, Western blot of protein extracted from an ITA. The 3 NOS antibodies and the $\beta$-actin antibody all stained protein bands at the expected molecular weights (not indicated). eNOS, endothelial nitric oxide synthase; iNOS, inducible nitric oxide synthase. C, Normalized (to $\beta$-actin) densities of the bands for the 3 isoforms of NOS in ITAs from 13 patients. Data are presented as means \pm standard error (eNOS vs nNOS: $P=.08$; eNOS vs iNOS: $\boldsymbol{P}=.70$; and $\mathrm{nNOS}$ vs iNOS: $\boldsymbol{P}=$ .12). D, Normalized densities of nNOS bands for the ITAs and SVs from 10 patients. Data are presented as means \pm standard error $(P=0.19)$. dures and reagents from Vectastain $\mathrm{ABC}$ Kits to attach a secondary antibody (and then peroxidase) to the primary antibody and a DAB kit to produce brown stain from the peroxidase (kits were from Vector Laboratories, Burlingame, Calif). The secondary antibody in the $\mathrm{ABC}$ kits contained biotinylated secondary antibody raised in horse against mouse $\mathrm{IgG}$ or in goat against rabbit IgG, depending on which primary antibody was being used. Negative controls, in which primary antibody was omitted from the PBS used for the overnight incubation (see above), showed that the secondary antibody and peroxidase did not bind to anything other than the primary antibody (Figures $2, D$, and $3, D$ ). Hematoxylin and Scott's blue were used for background staining.

\section{Data Analysis}

Western blot normalized densities were expressed as means \pm standard error. The 3 means for the isoforms of NOS were compared by using repeated-measures analysis of variance with Bonferroni adjustments for pairwise 2-tailed comparisons, and the 2 means for nNOS in ITAs and SVs were compared by using the paired 2-tailed $t$ test. Exact $P$ values are reported.

\section{Results}

\section{RT-PCR Studies}

An agarose gel of the products of a PCR carried out on cDNA from an ITA and an SV by using nNOS primers is shown in Figure 1, A. The primers used generate a 161-bp product from nNOS cDNA; it is clear from the bands seen on the gel at $161 \mathrm{bp}$ that nNOS mRNA is expressed in both the ITA and the SV. The smaller-sized bands are primer dimer. Comparable results were obtained with primers for iNOS and eNOS, indicating that all 3 NOS mRNA isoforms are expressed in both ITAs and SVs.

\section{Western Blot Studies}

Western blots of the protein extracted from the ITA of a patient are shown in Figure $1, B$. In this patient the density of the iNOS band was less than the density of the eNOS and nNOS bands, whereas in some other patients it was greater. The densities of the NOS bands on the Western blots were normalized to the density of the $\beta$-actin bands in the same lane. The bar graph in Figure 1, $C$, compares the average normalized band density of each isoform of NOS in the ITAs from 13 patients, and Table 1 gives the numeric values. From the $P$ values in Table 1, it can be seen that there is a $92 \%$ probability that the normalized average density for the nNOS band would be greater than the average for the eNOS band if we were to test the ITAs of hundreds of patients. Figure 1, $D$, shows the average normalized amounts of 


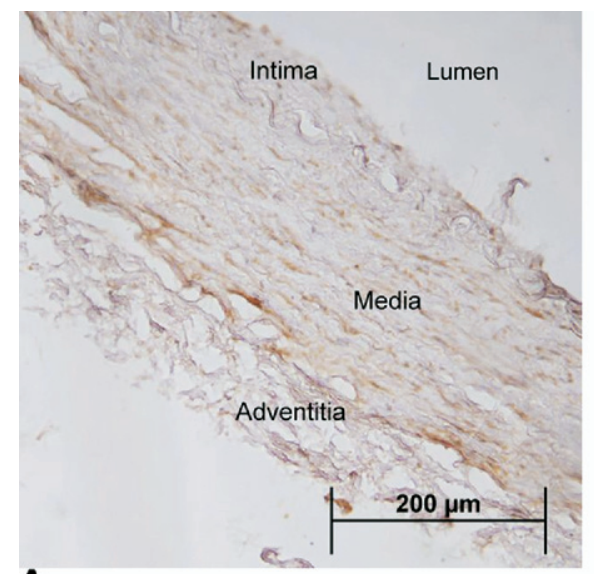

A

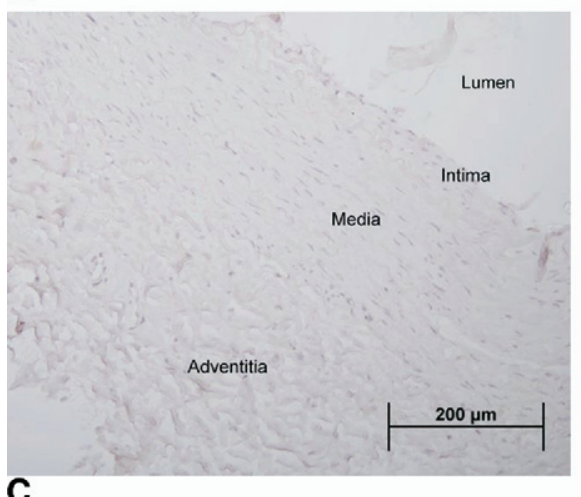

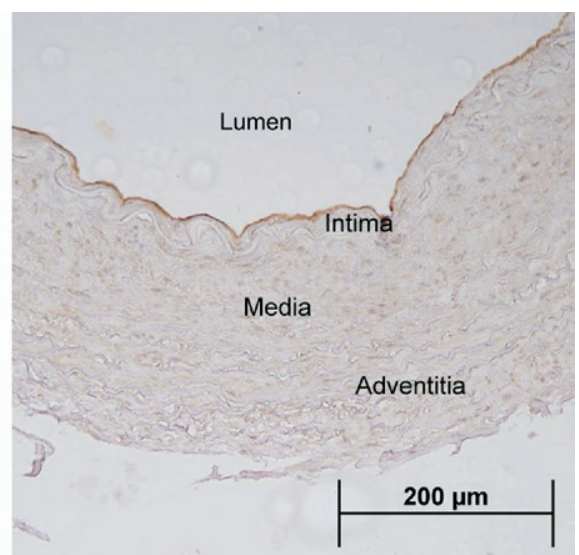

B

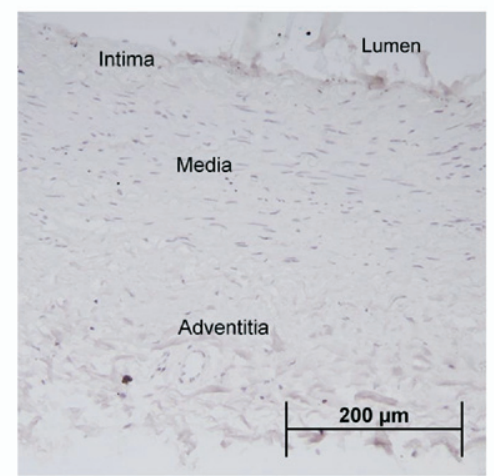

D
Figure 2. Nitric oxide synthase (NOS) immunohistochemistry of transverse sections of the internal thoracic artery (ITA) from one patient. The ABC peroxidase method was used to stain brown the sites where the primary antibody binds. A, Primary antibody specific for neuronal NOS (nNOS). B, Primary antibody specific for endothelial NOS (eNOS). C, Primary antibody specific for inducible NOS (iNOS). D, Negative control.
nNOS in the ITAs and SVs from 10 patients. The means \pm standard errors were as follows: $0.60 \pm 0.11$ for ITAs and $0.67 \pm 0.12$ for SVs. The paired $t$ test for comparison of the average amount of nNOS in ITAs (relative to $\beta$-actin) versus the corresponding amount in SVs yielded a $P$ value of .19. This is usually considered nonsignificant, although there is an $81 \%$ probability that the average amount of nNOS in the SV is greater than in the ITA.

\section{Immunohistochemistry Results}

There was considerable variation among patients in the amounts of each NOS isoform found in the ITA samples by

Table 1. Normalized density of the Western blot bands for the 3 isoforms of nitric oxide synthase in the human internal thoracic artery

\begin{tabular}{lccc}
\hline & eNOS & nNOS & iNOS \\
\hline Mean & 0.45 & 0.53 & 0.41 \\
SE & 0.08 & 0.06 & 0.07
\end{tabular}

eNOS vs nNOS: $P=.08$; eNOS vs iNOS: $P=.70$; nNOS vs iNOS: $P=.12$ ( $\mathrm{n}=13$ ). $P$ values are defined as the probability that the observed difference is due to chance selection of the sample. eNOS, Endothelial nitric oxide synthase (NOS III); nNOS, neuronal NOS (NOS I); iNOS, inducible NOS (NOS II); $S E$, standard error of the mean. means of Western blotting, and similar variations were found with immunohistochemistry. Figure 2 shows the expression of each of the 3 isoforms of NOS in an ITA from a patient with a relatively low amount of nNOS in smooth muscle (Figure 2, $A$ ) and no discernible iNOS (Figure 2, C). Some nNOS staining can also be seen in the endothelial cells. In this patient there was an appreciable amount of eNOS in the smooth muscle cells, and eNOS staining was very strong in the endothelium, as expected (Figure 2, B). Figure 3 shows sections of the ITA from a patient showing a large amount of nNOS in the smooth muscle cells (Figure 3, A). There was also some nNOS in the endothelial cells of this artery. The ITA from the same patient showed some eNOS in the smooth muscle cells, but a much stronger staining for eNOS was seen in the endothelium (Figure $3, B$ ). Staining for iNOS was seen in the ITA from this patient in both smooth muscle (media) and endothelium (Figure $3, C$ ). The $\mathrm{SV}$ of this same patient shows abundant staining for nNOS in the smooth muscle cells (Figure 3,E), and there was also a little eNOS in the media (Figure 3, $F$ ).

\section{Discussion}

Both iNOS and eNOS proteins have been measured with Western blotting in human ITAs. ${ }^{8}$ To our knowledge, however, nNOS protein has not previously been measured in 

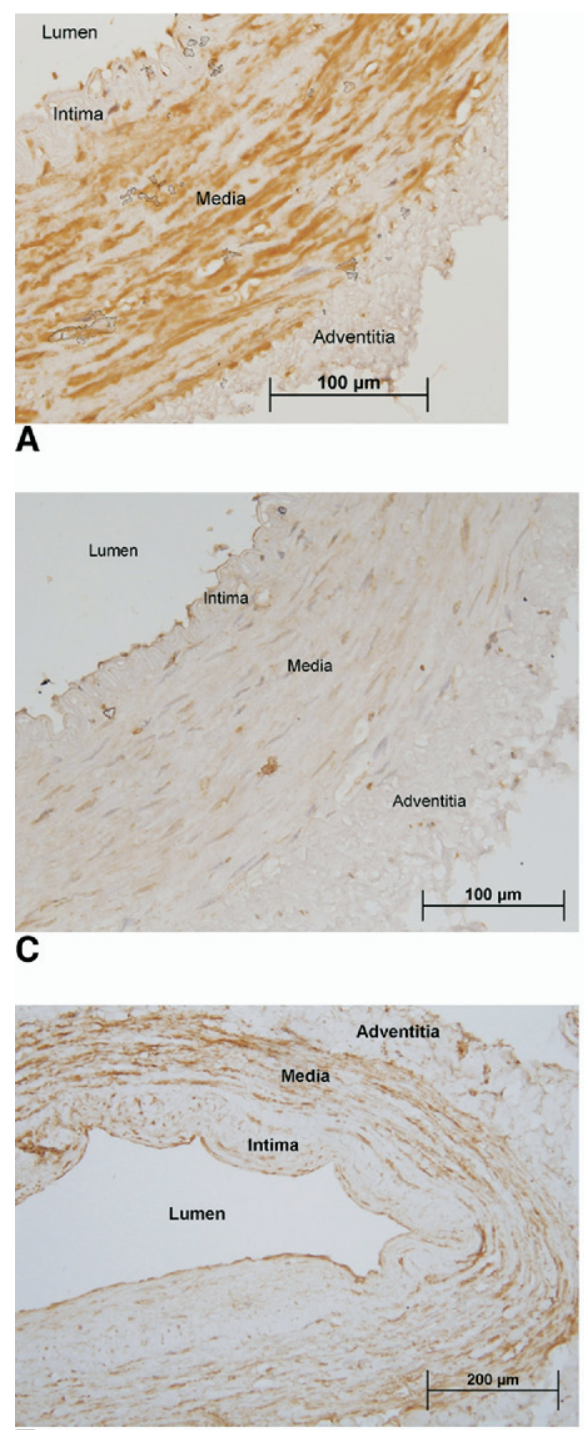

E

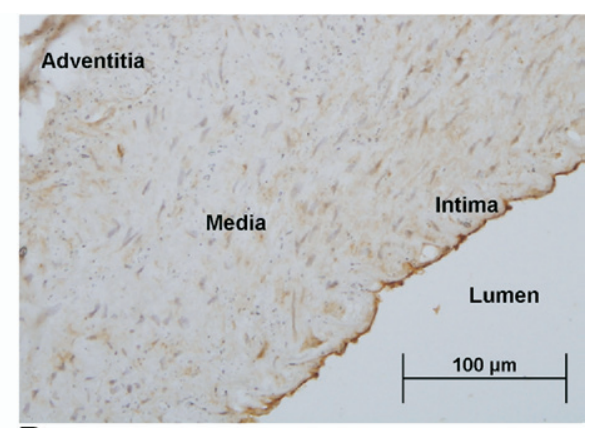

B

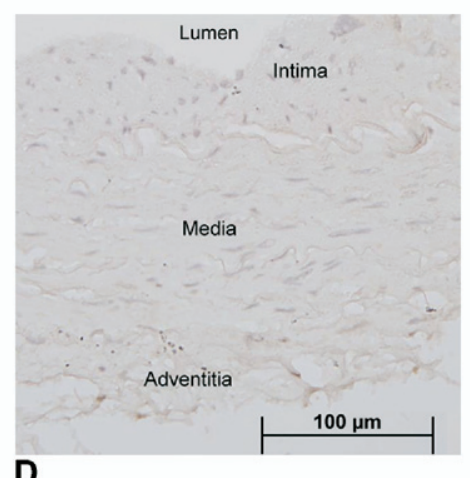

D

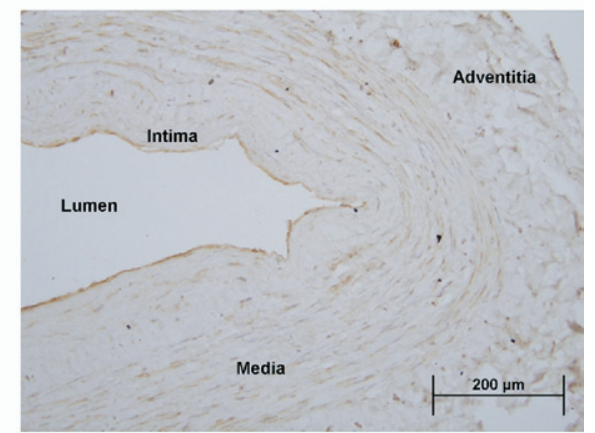

$\mathbf{F}$
Figure 3. Another patient in whom staining for neuronal nitric oxide synthase (nNOS) was more prominent. A, Internal thoracic artery (ITA) stained for nNOS. B, ITA stained for endothelial NOS (eNOS). C, ITA stained for inducible NOS (iNOS). D, ITA negative control. E, saphenous vein (SV) stained for nNOS. F, SV stained for eNOS.
ITAs. Neuronal NOS has been detected, by means of immunofluorescence, in the human external thoracic artery and some other muscular human arteries, where it was seen in both smooth muscle cells and endothelial cells. ${ }^{7}$ Similar to the present studies, these same investigators also observed a weak expression of eNOS in smooth muscle cells of the external thoracic artery. ${ }^{7}$ An immunohistochemistry study of samples of normal and atherosclerotic human aorta found that nNOS was expressed in the atherosclerotic vessels but not in normal vessels. ${ }^{9}$ The nNOS in the atherosclerotic aorta was associated with endothelial and mesenchymal cells in the intima. ${ }^{9}$ A recent review discusses studies that suggest that nNOS is inducible and that nNOS plays a protective role in atherosclerosis. ${ }^{10}$ In the human SV nNOS has been seen, with immunohistochemistry, in the media, as well as in adventitial perivascular nerves. ${ }^{11}$
Smooth muscle cells of the common carotid artery of the rat express nNOS protein, and Western blots show that arteries from hypertensive rats (SHR) contain more nNOS than do arteries from normal rats (WKY). ${ }^{6}$ Our Western blot data, which indicate that there might be as much nNOS protein as there is eNOS protein in the ITAs of hypertensive patients, suggest the possibility that under some conditions nNOS might be able to produce as much NO as eNOS. Both eNOS and nNOS are $\mathrm{Ca}^{2+}$-activated enzymes. In endothelial cells acetylcholine or bradykinin can trigger an increase in intracellular $\mathrm{Ca}^{2+}$ levels, which then activates eNOS; the resulting NO diffuses to and relaxes the smooth muscle cells. In vascular smooth muscle cells norepinephrine can trigger an increased intracellular $\mathrm{Ca}^{2+}$ concentration, which then contracts the cells, but if nNOS is present in the cells, the increased $\mathrm{Ca}^{2+}$ concentration might also result in the gen- 
eration of NO, which could moderate the contraction caused by the norepinephrine. Thus the increased nNOS levels found in the arteries of hypertensive rats might be an adaptive response to counter the increased blood pressure. More experiments are necessary to see whether this might also be true in human subjects.

Coronary artery bypass grafts using the SV do not remain patent for as long as grafts using the ITA. For example, in a study of 2127 coronary artery bypass grafts, only $32 \%$ of the SV grafts were still patent 15 years after the operation, whereas 96\% of the grafts using the left ITA were still patent after 15 years. ${ }^{12}$ Remainder segments of human ITAs have a higher basal level of NO production than segments of SVs, and furthermore, the duration of NO release after stimulation with bradykinin is longer with ITAs than with SVs. ${ }^{13}$ Also, in response to vascular endothelial growth factor, the ITA releases more NO than does the SV. ${ }^{14}$ The decreased release of $\mathrm{NO}$ in the SV might be due to the low or absent expression of eNOS in the smooth muscle cells of the human SV in contrast to a prominent expression of eNOS in the smooth muscle cells of the ITA. ${ }^{5}$ We observed some immunostaining of eNOS in the smooth muscle of both the ITA and the SV, but it was less intense than the nNOS staining. Also, it has been reported that there is less eNOS protein in the endothelial cells of the SV than in the ITA. ${ }^{15}$ The authors of all of the latter studies suggested that a lower NOS activity in SVs than in ITAs might explain why SV grafts do not last as long as ITA grafts. ${ }^{5,13-15}$ With Western blotting, we did not find less nNOS in SVs compared with ITAs. Thus our hypothesis that there is more nNOS in the ITA than in the SV was not supported, eliminating this as a possible explanation of the shorter lifespan of SV grafts.

Our finding of no major difference in the Western blot normalized band density for nNOS compared with eNOS suggests that there might be as much or more nNOS as there is eNOS protein in the human ITA. Thus, potentially, in some circumstances, nNOS might generate as much $\mathrm{NO}$ as does eNOS, giving nNOS a significant role in this artery. In the endothelium-denuded bovine carotid artery, physiologically active amounts of NO are generated by nNOS in the smooth muscle cells. ${ }^{16}$ Further experiments will clarify the role of nNOS in the ITA and might provide the basis for new treatments of atherosclerosis. Although our results clearly show that a substantial amount of nNOS protein is present, we should point out that because a different primary antibody must be used for each isoform, the band density might not be proportional to the amount of protein. This is because the binding efficiencies of antibodies can vary, and the amount of secondary antibody that binds to a particular primary antibody can also vary. On the other hand, our comparison of the amount of nNOS in the ITA with the amount in the SV is on firmer ground because we used the same nNOS primary antibody for all of the experiments.
We conclude that nNOS is expressed in the smooth muscle cells of both human ITAs and SVs. Neuronal NOS might play a significant role in relaxing the ITA because there can be as much nNOS in the ITA as there is eNOS Further experiments are needed to explore this possibility. Also, because there is little, if any, difference between the ITA and the SV in the amount of nNOS expressed, differences in nNOS expression apparently do not contribute to the difference in the longevity of grafts with these 2 vessels.

We thank Soh-Bee Yeo, Wan-Theng Sing, and Wendy C.-N. Chua for their expert technical assistance.

\section{References}

1. Furchgott RF, Zawadzki JV. The obligatory role of endothelial cells in the relaxation of arterial smooth muscle by acetylcholine. Nature. 1980;288:373-6.

2. Palmer RM, Ferrige AG, Moncada S. Nitric-oxide release accounts for the biological activity of endothelium-derived relaxing factor. Nature 1987;327:524-6.

3. Papapetropoulos A, Rudic RD, Sessa WC. Molecular control of nitric oxide synthases in the cardiovascular system. Cardiovasc Res. 1999 43:509-20.

4. Alderton WK, Cooper CE, Knowles RG. Nitric oxide synthase: structure, function and inhibition. Biochem J. 2001;357:593-615.

5. Gaudino M, Toesca A, Maggiano N, Pragliola C, Possati G. Localization of nitric oxide synthase type III in the internal thoracic and radial arteries and the great saphenous vein: a comparative immunohistochemical study. J Thorac Cardiovasc Surg. 2003;125:1510-5.

6. Boulanger CM, Heymes C, Benessiano J, Geske RS, Lévy BI, Vanhouttte PM. Neuronal nitric oxide synthase is expressed in rat vascular smooth muscle cells. Circ Res. 1998;83:1271-8.

7. Buchwalow IB, Podzuweit T, Böcker W, Samoilova VE, Thomas S, Wellner M, et al. Vascular smooth muscle and nitric oxide synthase. FASEB J. 2002;16:500-8.

8. Okon EB, Chung AWY, Rauniyar P, Padilla E, Tejerina T, McManus $\mathrm{BM}$, et al. Compromised arterial function in human type 2 diabetic patients. Diabetes. 2005;54:2415-23.

9. Wilcox JN, Subramanian RR, Sundell CL, Tracey WR, Pollock JS, Harrison DG, et al. Expression of multiple isoforms of nitric oxide synthase in normal and atherosclerotic vessels. Arterioscler Thromb Vasc Biol. 1997; 17:2479-88.

10. Tsutsui M. Neuronal nitric oxide synthase as a novel anti-atherogenic factor. J Atheroscler Thromb. 2004;11:41-8.

11. Tsui JC, Souza DSR, Filbey D, Karlsson MG, Dashwood MR. Localization of nitric oxide synthase in saphenous vein grafts harvested with a novel "no-touch" technique: potential role of nitric oxide contribution to improved early graft patency rates. J Vasc Surg. 2002;35:356-62.

12. Tatoulis J, Buxton BF, Fuller JA. Patencies of 2,127 arterial to coronary conduits over 15 years. Ann Thorac Surg. 2004;77:93-101.

13. Liu Z-G, Ge Z-D, He G-W. Difference in endothelium-derived hyperpolarizing factor-mediated hyperpolarization and nitric oxide release between human internal mammary artery and saphenous vein. Circulation. 2000;102(suppl III):III296-301.

14. Broeders MAW, Doevendans PA, Maessen JG, Gorsel EV, Egbrink MGAO, Daemen MJAP, et al. The human internal thoracic artery releases more nitric oxide in response to vascular endothelial growth factor than the human saphenous vein. J Thorac Cardiovasc Surg. 2001;122:305-9.

15. Zulli A, Hare DL, Horrigan M, Buxton BF. The resistance of the IMA to atherosclerosis might be associated with its higher eNOS and ET-A receptor immunoreactivity. Arterioscler Thromb Vasc Biol. 2003;23: 1308.

16. Brophy CM, Knoepp L, Xin J, Pollock JS. Functional expression of NOS 1 in vascular smooth muscle. Am J Physiol Heart Circ Physiol. 2000;278:H991-7. 